\title{
Design and Deployment of Testbed for Experimental Sensor Network Research
}

\author{
Zeeshan Hameed Mir, Hyeon Park, Young Bag Moon, \\ Nae Soo Kim, and Cheol Sig Pyo \\ USN/IoT Convergence Research Division, \\ Electronics \& Telecommunications Research Institute (ETRI), Republic of Korea \\ \{zhmir, hpark, moonyb, nskim, cspyo\} @etri.re.kr
}

\begin{abstract}
This paper presents the Ubiquitous Sensor Network (USN) testbed, which is deployed at Electronics and Telecommunications Research Institute (ETRI), in Republic of Korea. USN testbed is a state-of-the-art sensor network experimental facility composed of various components. It includes heterogeneous sensor nodes with variety of sensing module ranging from ultralow power to high performance hardware platforms, a scalable and reliable Ethernet based wired backplane network to program, interact with and receive/send data from/to the sensor nodes, an easily extendable application software suite which glues and drive everything in a user friendly manner, and an indoor and outdoor deployment space offering various environmental characteristics. We also included two case studies where researchers have put USN testbed into practice for experimental evaluation of algorithms and networking protocols. The case studies cover routing and clustering protocol in wireless sensor networks.
\end{abstract}

Keywords: Wireless Sensor Network, Experimental Testbed, Heterogeneous.

\section{$1 \quad$ Introduction}

For the last few years the importance of sensor network testbed [1] [2] [3] [4] [5] [6] [7] [8] has been continuously on the rise. Researchers are placing a considerable emphasis on the testbed infrastructure to validate theoretical system models as well as to evaluate performances of various sensor network applications. Testbed are also necessary to design, develop, and evaluate algorithms, protocols, and hardware platforms for the sensor network. There have been number of testbeds built to answer the limitations of simulation environment. However, most of these attempts tend to integrate several individual sensor nodes into a common infrastructure to achieve either certain application specific goals or a subset of the overall design objectives.

Sensor nodes must support various sensing modalities to cater diverse requirements impose by wide range of application scenarios. Moreover, the hierarchical organization of most of the sensor network applications demands sensor nodes to be built according to different capabilities at each successive level of the hierarchy. 


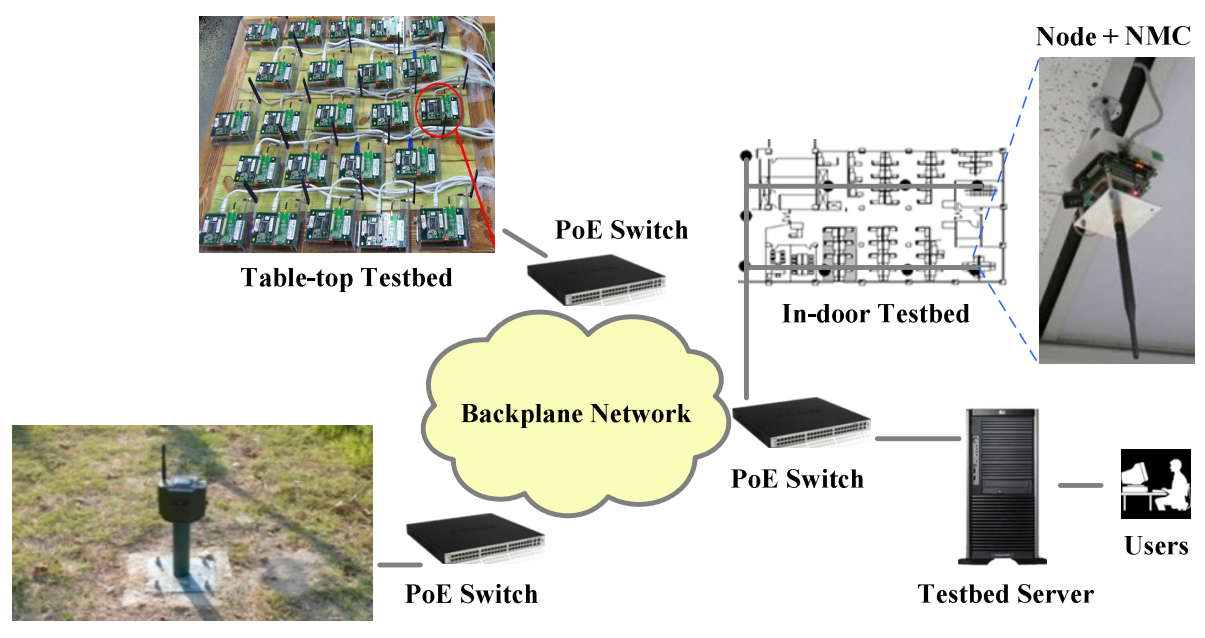

Out-door Testbed

Fig. 1. Main components of the USN testbed

The backplane must be a reliable and scalable infrastructure for node deployment, configuration and monitoring which is vital to any testbed. It is also necessary for the backplane network to operate without causing interference with the experiment itself. The testbed management software which glues and drive everything must be user friendly and easily extendable. Finally, the deployment space of the testbed must provide a wide range of environmental characteristics and propagation attributes in the form of both indoor and outdoor testbed.

There are two main contributions of this paper. Firstly, we describe Ubiquitous Sensor Network (USN) testbed architecture and its main components. USN testbed is a state-of-the-art sensor network experimental facility which is located within the premises of Electronics and Telecommunication Research Institute, Republic of Korea. USN testbed is composed of heterogeneous sensor nodes, an Ethernet based backplane [1] for experiment data collection and system monitoring, a versatile application software suite that allows users to interact with the testbed in an intuitive manner. The USN testbed deployment space not only covers indoor and outdoor environments but it can easily be extended for table-top/bench-top experiments as well. The second contribution is to discuss two case studies, where researchers have put USN testbed into practice for the implementation and testing of sensor network protocols. To demonstrate the different functionalities of the testbed we shared our experiences on routing, and clustering protocols in wireless sensor networks.

The remainder of the paper is organized as follows. In Section 2, we describe the overall architecture and main components of the USN testbed. In Section 3, we discuss in details each of the constituent component namely the deployment space, backplane network, sensor nodes, and the application software suite. In Section 4, we present two case studies where researchers have implemented and tested various protocols and applications for wireless sensor network using the USN testbed. Finally, in Section 5 we conclude this paper. 


\section{Ubiquitous Sensor Network (USN) Testbed: An Overview}

The USN testbad architecture consists of six main components, (1) Ethernet based backplane network, (2) Network Management Card (NMC), (3) Power over Ethernet (PoE) switches, (4) Testbed server, (5) Users, and lastly (6) Sensor nodes. Fig. 1 illustrates the main components of the USN testbed

1. Central to the USN testbed is the Ethernet based backplane network for collecting experimental results and other information. This wired medium is designed to connect testbed server and the NMC via PoE switches.

2. Network Management Card (NMC) acts as a middle-agent between the testbed server and the sensor node. NMC transfers the control signals/data from the testbed server to the sensor node. Likewise, it can also operate to get the feedback from a node to the testbed server. It consists of two layers, main board and interface communication board. The main board consists of micro-controller unit (MCU), memory, power supply and ISP (for program download), USB and JTAG. The interface communication board consists of CC2420 chipset, RS232 and LAN board.

3. Power over Ethernet (PoE) switch is used to supply power to operate the sensor node via NMC and collect data on the Ethernet cabling.

4. The testbed server is used to manage information regarding NMC, sensor nodes, users etc. The testbed server transfers the control commands to the NMC and sends resultant feedback via Ethernet network. The testbed server is designed to support all the required functions and information transfer to the users.

5. USN testbed supports multiple users running sensor network applications. Each user configures nodes to use wireless channel orthogonal to those used by the other users of the testbed.

6. Sensor nodes support various requirements imposed by a wide array of sensor networking application scenarios.

\section{Ubiquitous Sensor Network (USN) Testbed: Main Components}

\subsection{The Deployment Space}

Currently, the indoor testbed infrastructure is installed at the $1^{\text {st }}$ and the $5^{\text {th }}$ floors of Building No. 12, at the Electronics and Telecommunications Research Institute (ETRI). However, in the past the indoor testbed was deployed at the Ground and $2^{\text {nd }}$ floors of Building No.7. The nodes at the outdoor testbed are located along the boundaries of soccer and baseball fields within the ETRI premises. Moreover, the architecture of USN testbed can easily be extended by deploying table-top testbed. Some of the main information is given as under.

- Number of nodes: The indoor and outdoor testbed has a provision of up to 40 and 100 nodes, respectively. 
- Intra-node spacing: Indoor testbed (One Side: $3 \mathrm{~m}$ and another side: $3.5 \mathrm{~m}$ ) and Outdoor testbed $(10 \mathrm{~m})$.

- Height above the floor $(8 \mathrm{~cm}) /$ ceiling $(2.5 \mathrm{~m}): 180$ degree rotation is possible for various node orientations (useful for studying antenna orientation and its effect on testbed physical environment).

\subsection{The Backplane Network}

USN testbed employs an Ethernet based backplane network. The use of wired connections allows following testbed functions in a non-intrusive manner without introducing additional interferences.

- Gather debugging information,

- Real-time performance evaluation statistics collection,

- Transmit command/response to/from sensor nodes,

- Node status check, ON/OFF, and parameter adjustment (like transmit power),

- Remotely downloading code via Network Management Card (NMC),

- Supply power to the sensor nodes.

Out of total $10 \mathrm{PoE}$ switches, five of those switches are allocated for nodes in the outdoor environment, while four are at $5^{\text {th }}$ floor and the last one is at $1^{\text {st }}$ floor of Building No. 12. Each of the PoE switch can have up to 24 ports thus enabling connection to 24 nodes via NMC.

\subsection{The Hardware Platform}

The sensor nodes follow layered architecture to support diverse requirements imposed by various types of applications. The basic sensor node is composed of different modules such as sensing, micro-controller, RF and power supply module interconnected via inter-module interfaces.

The basic sensor node sensing module captures variety of physical properties from the environment such as Passive Infrared (PIR), light, acoustic, temperature/humidity, magnetic, vibration sensors. The node uses low-power MSP430 micro-controller module for sensor data processing and to provide inter-module and other interfaces such as SPI, ADC, UART, GPIO etc. MSP430 micro-controller is equipped with MSP430F2618 [9], 16-bits processor with Flash (116kB+256kB)/RAM (8kB) memory and 8MB Serial Flash. The MSP430 module drives DC $+3.7 \mathrm{~V}$ to $+3.3 \mathrm{~V}$ from the power module to supply power to MSP430F2618, Serial Flash and other circuitry. The RF module is a $2.4 \mathrm{GHz}$ CC2420 [10] chipset enabling 10-250kbps for wireless communication. The sensor node is also equipped with SMA-connector in order to connect with a range of external antennas (both Omni-directional and Sectored-antenna). The nodes can be powered either by Power over Ethernet (PoE) connected via Network Management Card (NMC) or through batteries.

The hierarchical organization of most of the sensor networks demand sensor nodes to be built according to certain roles for example, basic, cluster-head and gateway nodes. The ultra-low power design of the basic sensor node supports lightweight medium access protocols, dynamic routing and self-organized networking. 
Table 1. The Basic, Cluster-head and Gateway node hardware platform description

\begin{tabular}{llll}
\hline Feature & Basic node & Cluster-head & Gateway \\
\hline MCUs (1) & 16Bit RISC/16Mhz & 16Bit RISC/16Mhz & 16 Bit RISC/16Mhz \\
MCUs (2) & - & 32Bit & Xscale/13-520MHz \\
\hline Communication range & $30 \mathrm{~m}$ & $100 \mathrm{~m}$ & $100 \mathrm{~m}-7 \mathrm{~km}$ \\
Power supply & Battery/PoE & Battery/PoE & Battery/PoE \\
\hline Frequency/Data rate & $2.45 \mathrm{GHz} /$ & $433 / 915 \mathrm{Mhz}, 2.4 \mathrm{GHz} /$ & $2.45 \mathrm{GHz} /$ \\
(RF 1) & $10-250 \mathrm{Kbps}$ & $40-250 \mathrm{Kbps}$ & $250 \mathrm{Kbps}$ \\
Frequency/Data rate & - & $2.4 \mathrm{GHz} /$ & CDMA/ \\
(RF 2) & - & $250 \mathrm{Kbps}$ & $370 \mathrm{Kbps}-2 \mathrm{Mbps}$ \\
\hline Sensing module & & PIR/30m & GPS \\
/ Sensing range & & Acoustic/50m & CDMA \\
OR & & Magnetometer/5m & USB \\
Interfaces & Seismic/5m & Ethernet \\
\hline
\end{tabular}

However, to execute more computation and resource intense tasks (like networkwide schedule computation) USN testbed features the cluster-head nodes. Clusterhead nodes include dual micro-controllers (MSP430 and PXA270) for low-power consumption and high performance. At the RF module end, it incorporates options like $433 / 915 \mathrm{MHz}$ and $2.4 \mathrm{GHz}$ frequency capable of supporting 40 to $250 \mathrm{kps}$ of data rates. The sensor module can be enhanced further to capture audio and visual information as well. Hybrid sensor signal processing is provided with both low-power processor (e.g., MSP430F2618) and high performance digital signal processor (e.g., TMS320F28335 DSP [11]). The applications for cluster-head node are multiple target detection, classification and tracking, surveillance and cluster management.

Finally, the role of a gateway node is to converge information from multiple heterogeneous sources either as the final destination or as an intermediate link towards the storage unit. For these purposes, the design of gateway incorporate features of basic and cluster-head nodes with some more that are specific to its own role. For example, it can have an XScale micro-controller, CDMA supporting $370 \mathrm{kbps}$ to $2 \mathrm{Mbps}$ data rates, USB, Ethernet, GPS and power connections. The potential target applications of the gateway node are remote communication (e.g., inter-networking with Multimedia Terminal Mobile (MTM), hybrid MAC protocols (e.g., fusing TDMA and CSMA), GPS-based localization etc. Table 1, summarizes the hardware platform description of basic, cluster-head and gateways nodes.

\subsection{The Software Management Tool}

The software management tool is actually an application software suite. It includes Graphical User Interfaces (GUIs) to, (1) Issue commands and views the final data and performance evaluation statistics. (2) Apply topology and communication protocol level parameter settings. (3) Download and program the sensor nodes independent of the underlying physical sensor network testbed and embedded operating system. Following are the main software management programs of the USN testbed. 


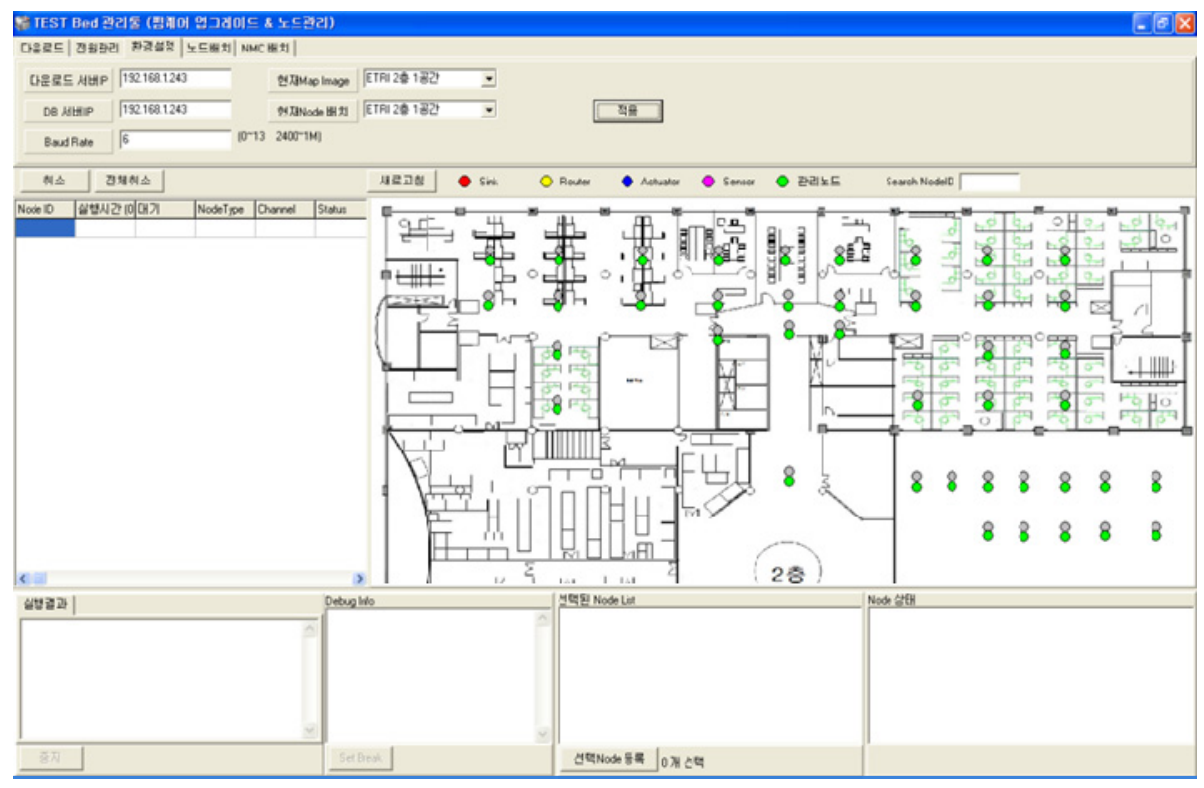

Fig. 2. Snapshot of USN Testbed Manager GUI

Server Program. A user must run the server program to remotely establish a connection with the USN testbed server. Once a successful connection is established the testbed server then configures the connection setting with the sensor node. These connection settings are directed by application software like USN Testbed Manager and the Testbed Tester. After completing the connection setting with the sensor node the testbed server sends the ID Query messages to the sensor nodes. The sensor nodes reply with the (IP, ID) pairs. The testbed server then stores and manages the UDP socket number and ID pair of the sensor nodes. The applications programs uses sensor node's ID to send commands and data to the testbed server. The testbed server lookup the ID-UDP socket number table and report the command to the corresponding sensor nodes via NMC.

Testbed Manager. This application software is a one stop solution for various tasks related with the experiment setup on USN testbed. The GUI is mainly composed of several tabs each offering a range of functions to its users. For example, a user can select nodes by choosing appropriate drop-down location options. The GUI node selection window allows selection/de-selection of a single node or a group of nodes with a simple drag and drop operation. Users then associate the NMC (with an IP address) to a particular node before downloading the source code binary. The source code binary is the compiled version generated separately using the IAR Compiler Software [12]. On successful or failed transmission of source code binary to the sensor node the appropriate result is displayed at the GUI of the user. Moreover, USN Testbed Manager also handles certain node management related function (like RESET). Fig. 2 illustrates the USN Testbed Manager software GUI. 


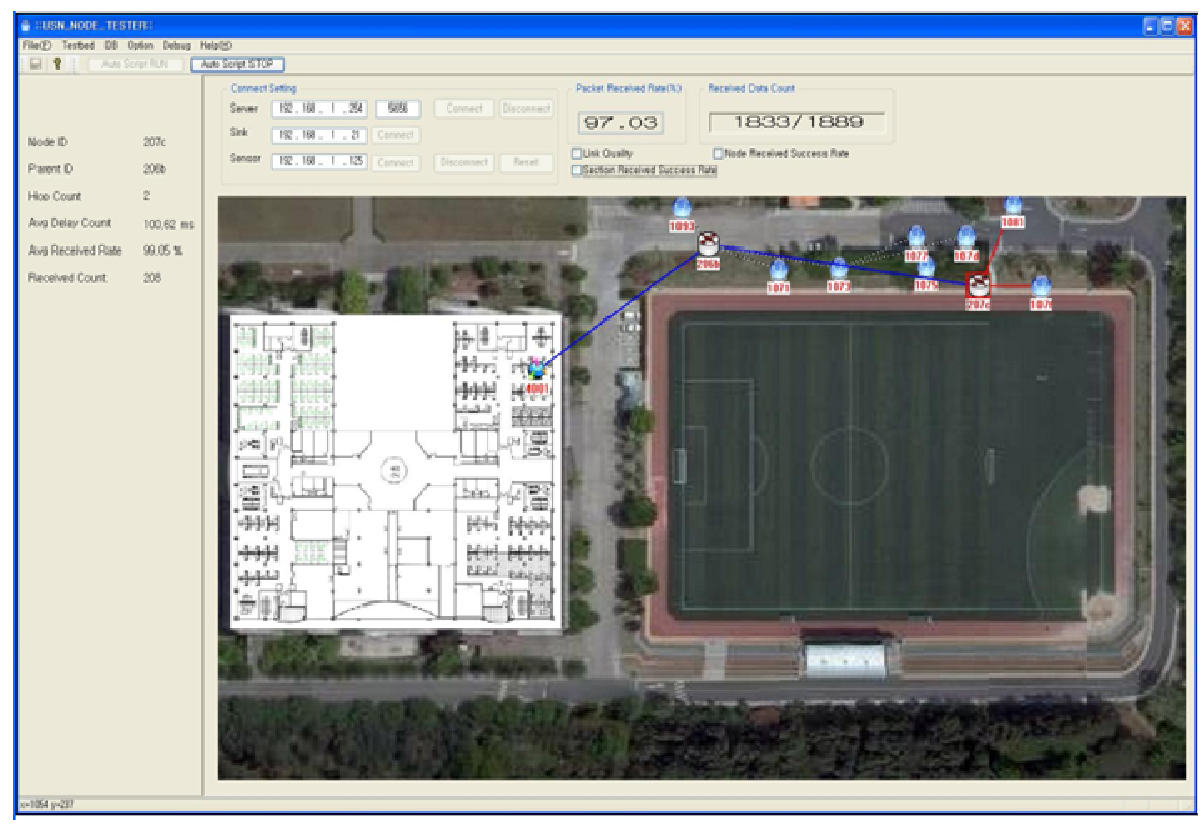

Fig. 3. Snapshot of USN Testbed Tester GUI

Terminal Program. The main purpose of this program is to send command to the sensor nodes and receive data from them at runtime. The interface allows the user to select a node (by the IP address of its attached NMC) and enter any string either in ASCII or hexadecimal format.

Testbed Tester. The actual experiments are executed using the USN Testbed Tester application software. This tool allows its users to configure the network topology, functioning of the nodes and communication protocol parameters based on the experiment requirements. The users can control the number of iterations a particular experiment can run with modified experiment parameters (like packet interval). The Testbed Tester collects and displays real-time performance evaluation statistics (like Packet Success Rate, End-to-End Delay etc.), nodes channel condition (like RSSI, LQI etc.) and sensing information (like light, temperature etc.). A user can also configure experiment to collection traces on per node basis. At the end of the experiments, the generated traces are stored in the spreadsheet software for later data analysis. The Testbed Tester program is easily extendable for various types of communication protocols and customized output generation. Fig. 3 illustrates the USN Testbed Tester software GUI.

\section{Ubiquitous Sensor Network (USN) Testbed: Case Studies}

The USN testbed has been used to study the performance and analysis of several control protocols including clustering, routing, cross-layer communication and 
medium access control (MAC) protocols. The testbed infrastructure has also been successfully utilized to deploy sensor network applications such as target detection and classification, surveillance and reconnaissance, pedestrian tracking to name a few.

\subsection{Case Study 1: MINT-Based Routing Protocol}

The USN testbed has been used to implement and test an enhanced version of the MINT (MINimum Transmission) [13] routing protocol. The enhanced version deals with real-time and reliable transfer of large amount of data (e.g. image) over the sensor network testbed. The application scenario that this protocol caters for include surveillance and reconnaissance.

The enhancement incorporates heterogeneity-aware TCP-like fragmentation mechanism to decide on the network-wide MTU (Minimum Transport Unit) size. Secondly, an improved packet retransmission scheme is proposed that adapt itself to the permissible loss rate sufficient enough to maintain prescribed image quality. Finally, the protocol uses expected number of transmissions along with neighbor status information to find more reliable paths. Extra features were added in the software management tools to visualize various types of node information such as node's neighbor table, details about averaged delivery ratio and delay time etc. It is also extended to take into account several of the new messages format. The sole purpose of these messages is to enable communication between the protocol and the USN Testbed Tester and the terminal program.

\subsection{Case Study 2: Static and Dynamic Clustering Protocol}

The USN testbed has been used to study the performance of static and dynamic clustering protocols. The static clustering algorithm designates few of the sensor nodes as the cluster-head during the network initialization process. While the dynamic clustering algorithm runs reactively as a result of certain event detection. In both cases the cluster-members will simply forward the data towards the cluster-head. The collected data is then aggregated at the cluster-head before it is sent to the sink node.

NanoQ-Plus [14], an ETRI proprietary operating system for embedded devices has been used to design, develop and load the program code on to the sensor nodes. The Testbed Tester and the terminal program were modified to allow specialized visualization of the results of the clustering algorithms. The sensor nodes take on different shapes and color based on their role in the network as a result of applying the clustering algorithms. Further details on the clustering protocols and test results can be found in [15].

\section{Conclusion}

This paper describes USN testbed, a state-of-the-art sensor network experimental facility. It is composed of several types sensor nodes deployed at multiple indoor and outdoor locations. The testbed provides an extendable Ethernet based backplane 
network so that real-time information can be transmitted without interfering and compromising the operation of the sensor network applications and protocol being tested. The USN testbed software component allows multiple users to manage and run separate experiments simultaneously. These software tools are used to program, send/receive messages to/from nodes and to gather real-time debugging information and statistical results of the experiments. We also presented two case studies where the scientists and researchers at ETRI have used the USN testbed for validating their protocols and applications. The presented case studies show the usability and the applicability of the USN testbed infrastructure to understand, apply and improve research and development in sensor networks.

Acknowledgments. This work was supported by the Dual Use Technology program of the DUTC, South Korea [Surveillance and Reconnaissance Sensor Network Development].

\section{References}

1. Crepaldi, R., et al.: The Design, Deployment, and Analysis of SignetLab: A Sensor Network Testbed and Interactive Management Tool. In: Proc. of TRIDENTCOM (2007)

2. Bapat, S., et al.: Chowkidar: A Health Monitor for Wireless Sensor Network Testbeds. In: Proc. of TRIDENTCOM (2007)

3. Werner-Allen, G., Swieskowski, P., Welsh, M.: Motelab: A Wireless Sensor Network Testbed. In: Proc. of IPSN (2005)

4. Arora, A., et al.: Kansei: A High-Fidelity Sensing Testbed. Proc. of IEEE Internet Computing 10, 35-47 (2006)

5. Gluhak, A., et al.: A survey on facilities for experimental internet of things research. Proc. of IEEE Communications Magazine 49(11), 58-67 (2011)

6. Rensfelt, O., et al.: SENSEI-A Flexible Testbed for Heterogeneous Wireless Sensor Networks. In: Proc. of TRIDENTCOM (2009)

7. Doddavenkatappa, M., Chan, M.C., Ananda, A.L.: Indriya: A Low-Cost, 3D Wireless Sensor Network Testbed. In: Korakis, T., Li, H., Tran-Gia, P., Park, H.-S. (eds.) TridentCom 2011. LNICST, vol. 90, pp. 302-316. Springer, Heidelberg (2012)

8. des Rosiers, C.B., Chelius, G., Fleury, E., Fraboulet, A., Gallais, A., Mitton, N., Noël, T.: SensLAB: Very Large Scale Open Wireless Sensor Network Testbed. In: Korakis, T., Li, H., Tran-Gia, P., Park, H.-S. (eds.) TridentCom 2011. LNICST, vol. 90, pp. 239-254. Springer, Heidelberg (2012)

9. DataSheet for (TI) MSP430F2618, http: / / focus . ti . com/

10. Datasheet for Chipcon (TI) CC2420 2.4 GHz IEEE 802.15.4/ZigBee RF Transceiver, http: / / www. chipcon. com

11. DataSheet for (TI) TMS320F28335, http: / / focus.ti.com/

12. IAR Systems, http: //www. iar.com/

13. Park, H., Ham, Y., Park, S., Woo, J., Lee, J.: Large Data Transport for Real-Time Services in Sensor Networks. In: Proc. of ComputationWorld (2009)

14. Park, S., Won Kim, J., Shin, K., Kim, D.: A Nano Operating System for Wireless Sensor Networks. In: Proc. of IEEE ICACT (2006)

15. Moon, Y., Kim, N., Lim, K., Jung, J., Ko, Y.: Efficient Cluster Head Election in Surveillance Reconnaissance Sensor Network. In: Proc. of ICWN 2010 - World Comp (2010) 